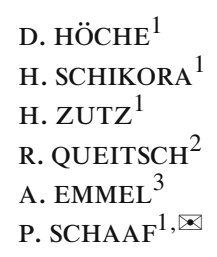

\title{
Microstructure of TiN coatings synthesized by direct pulsed Nd:YAG laser nitriding of titanium: Development of grain size, microstrain, and grain orientation
}

\footnotetext{
${ }^{1}$ Universität Göttingen, II. Physikalisches Institut, Friedrich-Hund-Platz 1, 37077 Göttingen, Germany

2 ARGES GmbH, Industrieplanung und Lasertechnik, Kirchplatz 4, 92507 Nabburg, Germany

${ }^{3}$ FH Amberg-Weiden, Lasertechnik, Kaiser-Wilhelm-Ring 23, 92224 Amberg, Germany
}

\begin{abstract}
Received: 25 October 2007/Accepted: 3 January 2008
Published online: 16 February 2008 • (C) The Author(s) 2008

ABSTRACT Pure titanium was irradiated by pulsed Nd:YAG laser irradiation in nitrogen atmosphere. As a result, nitrogen uptake and diffusion occurred and a TiN layer was synthesized at the titanium surface. These TiN coatings were analyzed by X-ray diffraction and the diffraction patterns were investigated in detail, in order to obtain more information about the physical processes during the coating formation. The diffraction peaks were fitted by Pearson VII profiles and the grain size and the microstrain were determined by the analysis of line broadening and peak shifts, using the Williamson-Hall and the Warren-Averbach formalisms. Additional single-line analyses were performed by means of the method of Langford and Keijser to obtain information about the preferred grain orientation and the texture development. The maximum grain size was about $100 \mathrm{~nm}$ and a corresponding average lattice strain of 0.002 was found. A relation between the treatment parameters and the coating properties, such as grain size and microstrain, can be shown. Thus, it was possible to determine optimal scan parameters for material processing and to establish the physical limits of the coating properties.
\end{abstract}

PACS 81.65.Lp; 81.15.Fg; 61.10.Nz; 68.55.Jk

\section{$1 \quad$ Introduction}

Nitriding and carburizing of metals are well known techniques to improve the tribological properties like hardness and wear resistance of technical surfaces [1]. As an example, plasma- and gas nitriding are established methods for surface treatment $[2,3]$. Alternatively, it is possible to treat the surface with laser radiation and to directly synthesize hard coatings [4-8]. Previous works have shown the successful synthesis of titanium nitride with laser irradiation [9-11]. Here, a Q-switched Nd:YAG laser was used for the nitriding treatment. The main advantages of this method are a reduction of the processing time and the negligible heat load for the workpiece. Stress, microstrain and inhomogeneity remain the main problems. Also the texture of cubic titanium nitride is a decisive factor for the technical quality of the coatings, as the

Fax:+49-551-39-4493, E-mail: pschaaf@uni-goettingen.de texture influences strain development and finally crack formation and propagation. Some investigations on texture and strain development in relation to the process parameters have been reported in the literature [12-14].

This work resolves the resulting grain size and strain development and relates them to laser treatment parameters like scan parameters. In this way, one can try to predict optimum treatment parameters for optimum coating properties, i.e. find parameters that minimize the lattice strain in order to reduce crack formation and propagation.

\section{$2 \quad$ Experiments \\ 2.1 Sample treatment}

Conventional $\alpha$-titanium (purity $>99.98 \%$ ) was used for the treatments. Titanium sheets of $1 \mathrm{~mm}$ thickness were cut into pieces of $15 \times 15 \mathrm{~mm}^{2}$ and used in the asreceived state without any further treatment.

For laser nitriding, the samples were placed in a chamber which was first evacuated and then filled with nitrogen (purity $99.999 \%$ ) to a pressure of $3 \times 10^{5} \mathrm{~Pa}$. The $\mathrm{Nd}$ :YAG laser irradiations were performed at the University of Applied Sciences in Amberg-Weiden, employing a Q-switched Nd:YAG laser in the first harmonic, i.e. the radiation had a wavelength of $532 \mathrm{~nm}$. At a repetition rate of $100 \mathrm{~Hz}$, the $6 \mathrm{~ns}$ (FWHM) pulses had a mean pulse energy of $40 \mathrm{~mJ}$. An optical scanner moved the laser beam across the whole sample surface in a meandering fashion over the samples, as shown in Fig. 1.

The quantitative parameter $\Sigma$ as defined in (1) is a dimensionless value for a parametric description of the process; it gives the total number of pulses hitting each surface area of the sample, and $n$ is the number of (identical) surface treatments for a given sample. The velocity $v$ of the lateral movement was varied between 10 and $50 \mathrm{~mm} / \mathrm{s}$. The energy density reaching the sample surface was changed by varying the spot size $d_{\mathrm{b}}(0.70$ to $1.15 \mathrm{~mm})$. The lateral shift $\delta$ between subsequent laser scan lines was varied from 0.05 to $0.20 \mathrm{~mm}$.

$\Sigma=n \frac{d_{\mathrm{b}}^{2} f_{\text {pulse }}}{v \delta}$.

The intense laser irradiation heats and melts the titanium surface and a plasma forms on top of the surface, resulting in the dissociation of nitrogen and its in-diffusion into the melt. 


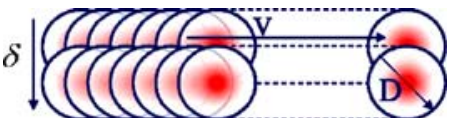

FIGURE 1 The meandering of the titanium samples is described by the lateral shift $\delta$, the scan velocity $v$ and the spot diameter $d_{\mathrm{b}}$. Together with the pulse frequency $f_{\text {pulse }}$ and the geometric parameters, the irradiation of each surface element is described quantitatively

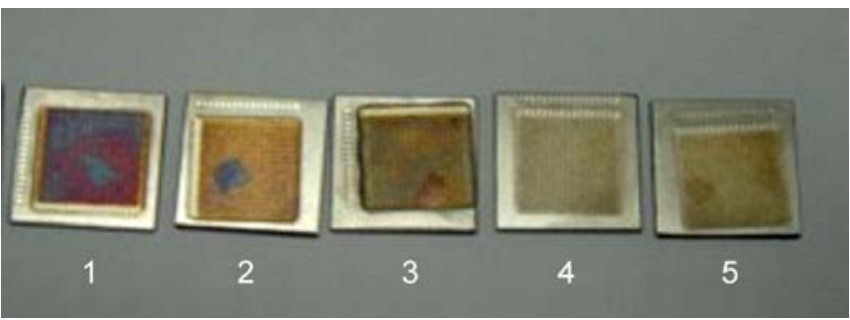

FIGURE 2 Photographs of the laser nitrided titanium samples. The scan parameters are given in Table 1

\begin{tabular}{rrcccc}
\hline No. & $n$ & $d_{\mathrm{b}}[\mathrm{mm}]$ & $\delta[\mathrm{mm}]$ & $v[\mathrm{~mm} / \mathrm{s}]$ & $\Sigma$ \\
\hline 1 & 2 & 1.00 & 0.10 & 30 & 66.7 \\
2 & 1 & 1.00 & 0.10 & 30 & 33.3 \\
3 & 1 & 1.00 & 0.05 & 30 & 66.7 \\
4 & 1 & 1.00 & 0.20 & 30 & 16.7 \\
5 & 1 & 1.00 & 0.15 & 30 & 22.3 \\
6 & 1 & 1.00 & 0.10 & 10 & 100.0 \\
7 & 1 & 1.00 & 0.10 & 20 & 50.0 \\
8 & 1 & 1.00 & 0.10 & 40 & 25.0 \\
9 & 1 & 1.00 & 0.10 & 50 & 20.0 \\
10 & 1 & 0.70 & 0.10 & 30 & 16.3 \\
11 & 1 & 0.85 & 0.10 & 30 & 24.1 \\
12 & 1 & 1.15 & 0.10 & 30 & 44.1 \\
\hline
\end{tabular}

TABLE 1 Scan parameters of the laser nitrided samples. For all the samples $f=100 \mathrm{~Hz}$ and $p=3 \mathrm{bar}$. The number of repeated (identical) treatments (scans) $n$, the spot diameter $d_{\mathrm{b}}$, lateral shift $\delta$ and overlap parameter $\Sigma$ are given

After laser irradiation, solidification begins and different TiN coatings develop. Some examples for the produced coatings are shown in Fig. 2; the treatment parameters are summarized in Table 1.

\subsection{Sample analysis}

The samples were analyzed by a range of methods [11], while the focus here will be on the results from X-ray diffraction (XRD). XRD was performed on a Bruker AXS D8 Advance diffractometer equipped with a $\mathrm{Cu} K_{\alpha}$ long-finefocus tube. The beam is collimated and compressed by a Göbel mirror and a $0.6 \mathrm{~mm}$ slit to a collimated beam of low divergence with dimensions of about $0.6 \mathrm{~mm}$ by $11 \mathrm{~mm}$. The sample is located at a distance of about $40 \mathrm{~cm}$. The exit beam was conditioned with a Soller slit assembly and a monochromator with $0.1 \mathrm{~mm}$ detector silt. All XRD patterns shown here were scanned in Bragg-Brentano geometry with a $2 \Theta$ range from $30^{\circ}$ to $100^{\circ}$ and a step size of $0.025^{\circ}$.

\section{Theory}

\subsection{The Ti-N system}

The equilibrium Ti-N phase diagram, the properties of titanium nitride and the solubility of nitrogen in tita- nium are well known [15]. The thermodynamic relationships and the effects on the melting behavior have also been described [16]. The composition dependent liquidus temperature, the solubility of 23 at. \% nitrogen in titanium without any formation of a stable phase and the variation of the lattice parameter as a function of the nitrogen content in $\delta-\mathrm{TiN}_{x}$ are important factors for the present investigations. According to Vegard's law [17], the latter relation is given by (2), based on the experimental data [18] for cubic TiN.

$a=4.1925 \AA+x 0.0467 \AA$.

\section{2}

\section{XRD peak analysis}

The measured XRD reflections $f_{\text {peak }}(x)$ are a sum of physical and instrumental influences, which can be treated as a convolution given in (3). Line broadening in XRD arises mainly due to three factors: the machine influence, the strain broadening and the effects based on finite grains (size effect).

$f_{\text {peak }}(x)=g_{\text {instr }}(x) \otimes h_{\text {phys }}(x)+b_{\text {bg }}(x)$,

$h_{\text {phys }}(x)=h_{\text {strain }}(x) \otimes h_{\text {size }}(x)$.

Instrumental effects $g_{\text {instr }}(x)$ include imperfect focusing, finite beam width and the $K_{\alpha_{1}}, K_{\alpha_{2}}$ problem. These effects can be removed by the Stokes correction [19], which is based on a Fourier analysis. In addition, a single $\mathrm{MgO}$ crystal was measured to resolve the instrument properties. For this purpose, the crystal was assumed to be perfect and the pure instrumental broadening was measured. The sample broadening arises due to lattice strain and finite grain size $h_{\text {phys }}(x)$ or it may result from variations in the lattice constant or grain boundary effects.

The diffraction spectra were evaluated using the program WinFit [20]. All the peaks were fitted by the split Pearson VII function as mentioned in [21]. This is a combination of Gaussian and Lorentzian (Cauchy) type peak profiles. For the peak width $B_{\text {peak }}$ of the peak broadening after instrumental correction, the following equation (4) is valid:

$$
\begin{array}{ll}
B_{\text {peak, } \mathrm{C}}=B_{\text {strain, } \mathrm{C}}+B_{\text {size } \mathrm{C}} & (\text { Cauchy profile }), \\
B_{\text {peak, } \mathrm{G}}^{2}=B_{\text {strain, } \mathrm{G}}^{2}+B_{\text {size, } \mathrm{G}}^{2} & (\text { Gaussian profile }) .
\end{array}
$$

As a result of the peak fitting, the ratios of Gaussian and Lorentzian broadening can be determined and used for the calculation of strain and grain size. Two common methods of X-ray diffraction line broadening analysis are the Williamson-Hall plot (WHP), based on the work of Scherrer and Wilson and the Warren-Averbach analysis (WA). There are further methods such as single [22] or double Voigt [23] analysis, where the single line analysis is used to measure the strain in the lattice planes. Here, an asymmetric Voigt function was fitted to the diffraction reflexes by means of the program Fityk [24]. The resulting Gaussian ratio can be used for the strain investigations.

3.2.1 Williamson-Hall plot (WHP). Scherrer [25] developed a first relation, as given in (5), to determine the grain size 
$D_{B}$ from X-ray diffraction peak profiles. This equation is still widely used as a first approximation for calculations.

$D_{B}=\frac{K \lambda}{B \cos \Theta}$.

$K$ is the Scherrer factor (usually close to unity), $\lambda$ is the wavelength of the radiation, $B$ is the peak width (FWHM) and $\Theta$ is the Bragg angle of the reflection of the corresponding $(h k l)$ plane. Later, Wilson [26] replaced $B$ by $\beta$, a volume weighted quantity. The integral width $\beta$ is defined as the ratio of the peak area $A$ and the peak intensity $I$ of the $(h k l)$ reflection: $\beta=A_{h k l} / I_{h k l} . \beta$ is only attributed to the finite grain size. For a broadening originating solely from strain effects, Wilson [27] gave a relationship between an apparent strain $\eta$ and the integral width $\beta$.

$\eta=\beta_{h k l} \cot \Theta=4 \varepsilon=4 \frac{a-a_{0}}{a_{0}}$,

where $\varepsilon$ is the maximum strain in the lattice parallel to the reflection planes. Furthermore, the lattice constant $a$ can be determined and the nitrogen content can be verified by means of Vegard's law.

In order to separate the effects of strain and grain size, Williamson and Hall [28] developed a method known as the Williamson-Hall plot (WHP):

$\beta=\left\{\frac{K \lambda}{D_{\beta} \cos \Theta}\right\}+4 \varepsilon \tan \Theta$.

Plotting $\frac{\beta \cos \Theta}{\lambda}$ versus $\frac{\sin \Theta}{\lambda}$ results in a linear fit and the slope $\eta$ and the intercept $K / D(\beta)$ can be determined. Actually, this method is only valid for pure Cauchy peaks, but as a first approximation the accuracy can be assumed sufficient for our purpose. As a clear disadvantage, only the maximum strain can be resolved.

3.2.2 Warren and Averbach (WA). A more accurate method was developed by Warren and Averbach [29]. They proposed that the peak broadening is a product of the Fourier coefficients of the size function $A_{\text {size }}(L, s)$ and the strain function $A_{\text {strain }}(L, s)$ :

$A(L, s)=A_{\text {size }}(L) \cdot A_{\text {strain }}(L, s)$,

where $s$ is the diffraction vector and $A_{\text {size }}$ is independent of the reflection order (i.e. independent of $s$ ). $L$ as given by (9)), is a distance normal to the $(h \mathrm{kl})$ reflection plane with the interplanar spacing $d_{\mathrm{WA}}$ and $n$ being an integer:

$L=\frac{n \lambda}{2\left(\sin \Theta_{2}-\sin \Theta_{1}\right)}=n d_{\mathrm{WA}}$.

$\Theta_{1,2}$ limits the range where every peak is measured. Further, it was shown in $[30,31]$ that the following equation (10) is valid:

$\ln A(L, s)=\ln A_{\text {size }}(L)-2 \pi^{2} s^{2} L^{2}\left\langle\varepsilon^{2}(L)\right\rangle$.

This function is independent of the reflection order. The mean square strain $\left\langle\varepsilon^{2}(L)\right\rangle$ will be determined in the $s$ direction. Thus, information about the strain not parallel to the sample surface is resolvable. By means of the diffraction vector $s, \ln A(L, s)$ can be plotted against $s^{2} a^{2}$ for several values of $L$. The intercept of this plot gives $\ln A_{\text {size }}(L)$ (grain size influence) and the slope gives the mean square strain $\left\langle\varepsilon^{2}(L)\right\rangle$.

Finally, Warren and Averbach [29] have shown that the intercept of the initial slope of the plot gives the average $L$ range and with (11) the surface weighted grain size distribution $f_{\text {dis }}(L)$.

$$
\left(\frac{\mathrm{d} A_{\text {size }}(L)}{\mathrm{d} L}\right)_{L \rightarrow 0}=-\frac{1}{D_{\text {size }}} .
$$

In the work of Wagner et al. [31-33], investigations of several materials like tungsten and aluminium are reported and the comparison of the different methods yielded similar results.

$f_{\text {dis }}(L) \propto \frac{\mathrm{d}^{2} A_{\text {size }}(L)}{\mathrm{d} L^{2}}$.

The method described here is implemented in the Winfit program [20]. An accurate description of this analysis is given in $[29,30,34]$.

3.2.3 Single line analysis and preferred orientation. In order to determine the strain in the ( $h k l)$ planes, Langford [23] proposed to use the Voigt function for peak fitting. Based on this work, Keijser [22] supposed that the Gaussian component of the line broadening only arises from the strain and the Lorentzian part only from the grain size. By means of (13) and the measured Gaussian integral width $\beta_{\mathrm{G}}$, it is easy to obtain the lattice strain.

$\varepsilon_{h k l}=\frac{\eta_{h k l}}{4}=\frac{\left(\beta_{\mathrm{G}}\right)_{h k l}}{4 \tan \Theta_{h k l}}=\frac{a_{h k l}-a_{0}}{a_{0}}$.

The actual orientation of the grains mainly depends on the Gibbs free energy of the phase planes. Theoretical studies by McKenzie et al. [35] showed that the preferred orientation depends on the minimum value of the overall energy in the planes. This energy is the sum of the surface energy $S_{h k l}$ and the energy in the stress field $U_{h k l}$. $S_{h k l}$ depends on the sublimation energy and the number of broken atom bonds [13]. However, the stress energy becomes important if it becomes larger than the surface energy.

\section{$4 \quad$ Results \\ 4.1 Grain size and strain}

Figure 3 presents an example of a $\Theta-2 \Theta$ scan of laser nitrided titanium. $\operatorname{TiN}_{x}$ is cubic $(F m 3 m, 225)$ and has a lattice constant of $a=4.24 \AA$ [36] for $x=1$. The virgin titanium is hexagonal $(P 63 / m m c, 194)$ and has lattice constants of $a=2.950 \AA$ and $c=4.682 \AA$ [37]. $\alpha$-Ti is still observed in the coatings. This is an indication that the coating is not homogeneous and that under-stoichiometric titanium nitride may be present. Due to diffusion and convection effects during the laser nitriding process, the nitrogen and phase distribution is not homogeneous throughout the coating.

Further, a texture parameter $\eta_{\mathrm{t}}$ is defined as the ratio of the (111) to (200) peak intensities, i.e. $\eta_{\mathrm{t}}=I(111) / I(200) \cdot \eta_{\mathrm{t}}=0$ 


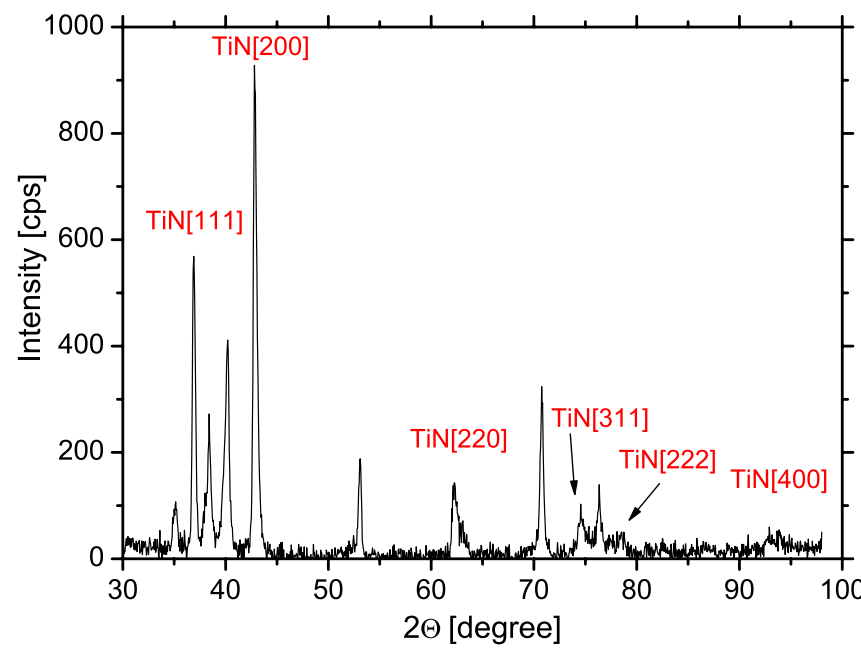

FIGURE 3 Full XRD spectrum in Bragg-Brentano geometry for sample 2

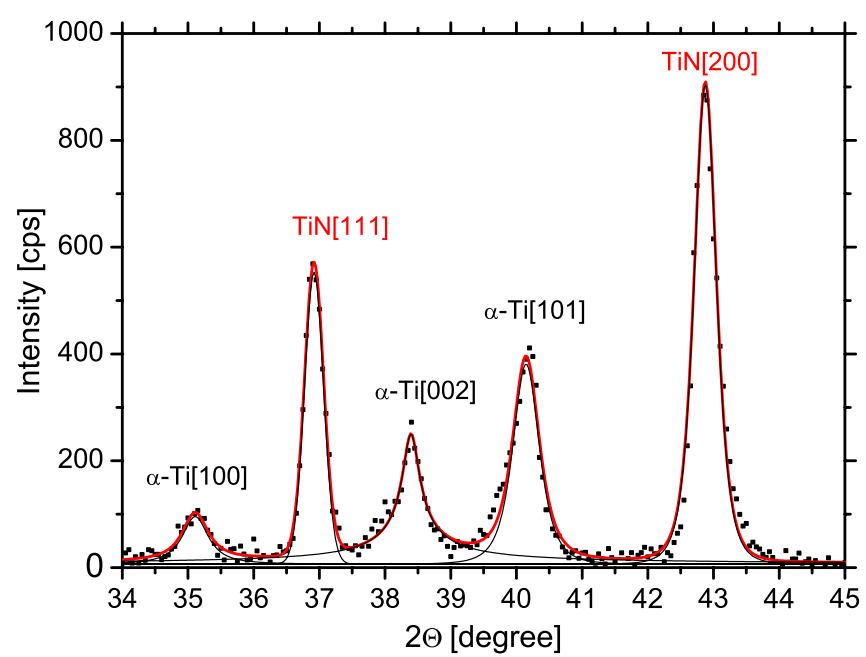

FIGURE 4 Detail of Fig. 3 in the range $34-45^{\circ}$, showing the Pearson VII peak fits

gives a perfect (200) texture, whereas $\eta_{\mathrm{t}}=0.72$ is obtained for a perfect polycrystal without any texture [9].

The results of the multipeak fits of this spectrum are presented in Figs. 4 and 5, and the fit results are summarized in Table 2 for all observable $(h \mathrm{kl})$ reflexions of TiN.

Based on the fit result given in Table 2, the strain and grain size have been derived by the WHP and WA formalisms as described before. Due to the used step size of $0.025^{\circ}$, the fitting error of $B$ was up to $5 \%$ and by error propagation the following results are subject to error limits of about $20 \%$. The step size limit of the machine is $0.001^{\circ}$. As a result, the error could be reduced to approximately $5 \%$ for the grain size values, but with a drastic increase in measuring time.

\begin{tabular}{lcccc}
\hline Peak & $2 \Theta$ [deg.] & $B$ [deg.] & $\beta$ [deg.] & $\beta_{G}$ [deg.] \\
\hline 111 & 36.893 & 0.331 & 0.362 & 0.054 \\
200 & 42.824 & 0.396 & 0.477 & 0.038 \\
220 & 62.164 & 0.564 & 0.770 & 0.064 \\
311 & 74.495 & 0.711 & 1.155 & $>0.1$ \\
\hline
\end{tabular}

TABLE 2 Results of the peak fits shown in Figs. 4 and 5 for all $(h \mathrm{kl})$ reflexes of TiN

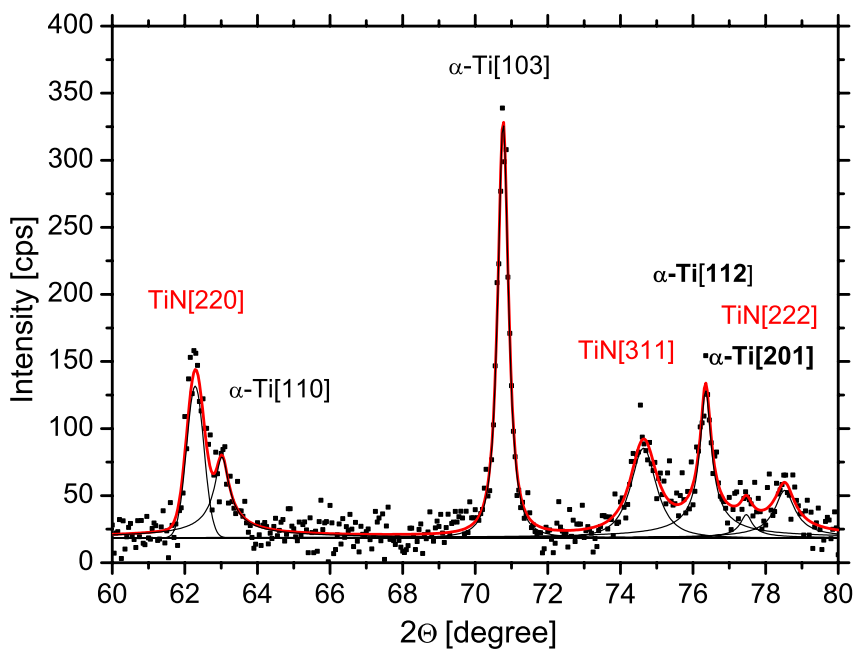

FIGURE 5 Detail of Fig. 3 in the range $60-80^{\circ}$, showing the Pearson VII fits

4.1.1 Influence of scan velocity. As an example, in Fig. 6 a WHP plot for different scan velocities is shown. The linear fitting used for the analysis is also depicted there. The results are summarized in Table 3.

It is observed that $\eta_{\mathrm{t}}$ increases with the scan velocity which means that the texture changes from (200) to (111) preference. As expected, the coating thickness $t-$ measured by cross section SEM [11] - decreases with increasing scan velocity.

The scan velocity is one of the most important parameters for the process. For high scan velocities, the strain increases and the grain size decreases. This is due to the shorter interaction time for higher velocities and also to the fact that the time and energy become too small for deep melting. In addition, less energy lowers the temperature so that no evaporation plasma occurs and, consequently, there is no plasma activation for an efficient nitrogen inward diffusion.

On the contrary, for low velocities other effects determine the coating properties. The high energy input leads to remelting effects and strong vaporization occurs. This causes more strain, lattice deformations and cracks.

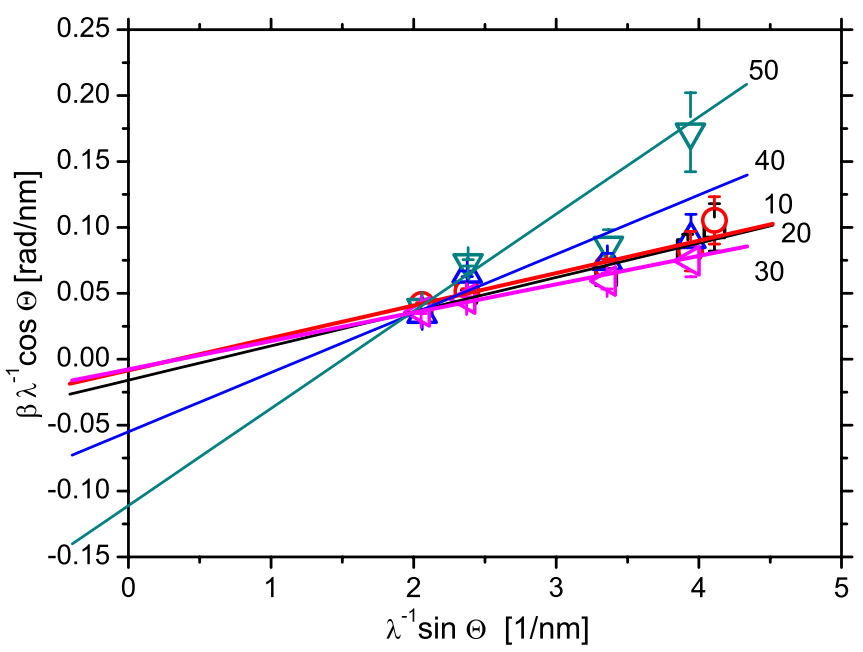

FIGURE 6 WHP plot for different scan velocities (given in $\mathrm{mm} / \mathrm{s}$ ). Detailed scan parameters are given in Table 1 (samples 2, 6-9) 


\begin{tabular}{lcrrrrrr}
\hline $\begin{array}{l}v \\
{[\mathrm{~mm} / \mathrm{s}]}\end{array}$ & $\eta_{\mathrm{t}}$ & $\Sigma$ & $\begin{array}{c}\varepsilon_{\mathrm{WHP}} \\
{[\%]}\end{array}$ & $\begin{array}{c}D_{\mathrm{WHP}} \\
{[\mathrm{nm}]}\end{array}$ & $\begin{array}{c}\varepsilon_{\mathrm{WA}} \\
{[\%]}\end{array}$ & $\begin{array}{c}D_{\mathrm{WA}} \\
{[\mathrm{nm}]}\end{array}$ & $\begin{array}{c}t \\
{[\mu \mathrm{m}]}\end{array}$ \\
\hline 10 & 0.424 & 100.0 & 0.650 & 62.9 & 0.221 & 27.6 & 1.44 \\
20 & 0.707 & 50.0 & 0.615 & 118.3 & 0.181 & 35.1 & 1.27 \\
30 & 0.732 & 33.3 & 0.535 & 126.4 & 0.201 & 53.8 & 1.21 \\
40 & 0.753 & 25.0 & 1.122 & 18.4 & 0.219 & 44.9 & 1.03 \\
50 & 0.754 & 20.0 & 1.842 & 9.0 & 0.323 & 29.5 & 0.80 \\
\hline
\end{tabular}

TABLE 3 Results of the XRD analyses: texture parameter $\eta_{\mathrm{t}}$, overlap $\Sigma$, strain $\varepsilon$ and grain size $D$ as obtained by the WHP and WA formalisms, and film thickness $t$ for different scan velocities $v\left(d_{\mathrm{b}}=1.00 \mathrm{~mm}, \delta=0.1 \mathrm{~mm}\right.$, $f=100 \mathrm{~Hz})$

There is an optimum scan velocity of 20 to $30 \mathrm{~mm} / \mathrm{s}$ as seen in Fig. 7. The local energy input is high enough for deep melting and nitrogen dissociation, but the convection and plasma effects do not create cracks. Furthermore, the average grain size (WA analysis) increases to a maximum of about $50 \mathrm{~nm}$ and the strain $\varepsilon$ is reduced to a minimum of about $2 \times 10^{-3}$. The nitrogen content $x$ ranges between 0.72 and 0.88 , calculated from the lattice constant and (2).

4.1.2 Influence of the spot diameter. The next parameter that was varied is the spot diameter. By changing the spot size, the energy density was varied. Table 4 shows the coating properties for different spot diameters $d_{\mathrm{b}}$. Similar effects with similar origins for crack development, strain distribution, and coating thickness are observed as for the case of the velocity change.

Optimal coating properties were found for a spot diameter of $1 \mathrm{~mm}$, where a nitrogen content of $x=0.84$ was derived. The WHP plot given in Fig. 8 shows a strong dependence

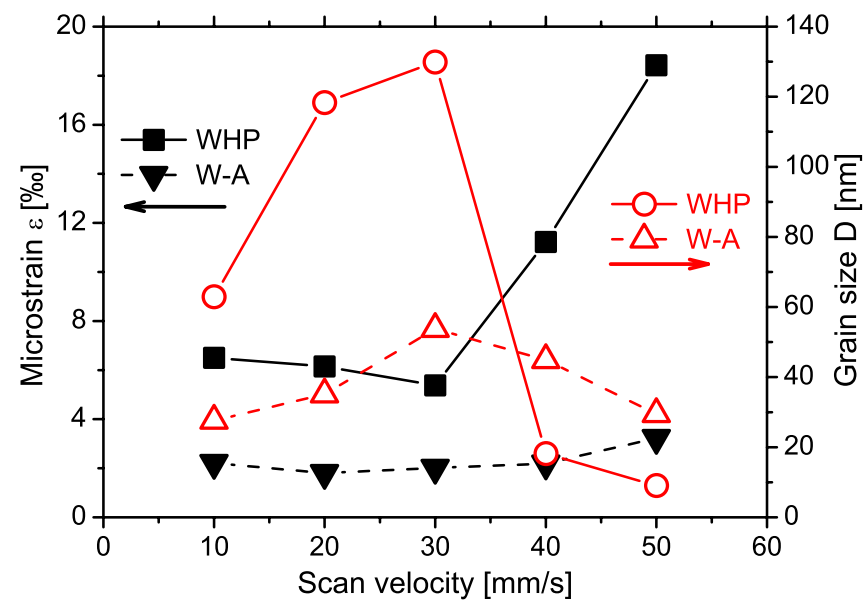

FIGURE 7 Derived strain and grain size as a function of the scan velocity

\begin{tabular}{lcccrccc}
\hline $\begin{array}{l}d_{\mathrm{b}} \\
{[\mathrm{mm}]}\end{array}$ & $\eta_{\mathrm{t}}$ & $\Sigma$ & $\begin{array}{c}\varepsilon_{\mathrm{WHP}} \\
{[\%]}\end{array}$ & $\begin{array}{c}D_{\mathrm{WHP}} \\
{[\mathrm{nm}]}\end{array}$ & $\begin{array}{c}\varepsilon_{\mathrm{WA}} \\
{[\%]}\end{array}$ & $\begin{array}{c}D_{\mathrm{WA}} \\
{[\mathrm{nm}]}\end{array}$ & $\begin{array}{c}t \\
{[\mu \mathrm{m}]}\end{array}$ \\
\hline 0.70 & 0.615 & 16.3 & 0.690 & 82.2 & 0.224 & 35.3 & 1.36 \\
0.85 & 0.695 & 24.1 & 0.740 & 77.1 & 0.244 & 40.8 & 1.29 \\
1.00 & 0.732 & 33.3 & 0.535 & 126.4 & 0.201 & 53.8 & 1.21 \\
1.15 & 0.841 & 44.1 & 1.015 & 36.3 & 0.248 & 30.6 & 1.18 \\
\hline
\end{tabular}

TABLE 4 Results of the XRD analyses: texture parameter $\eta_{\mathrm{t}}$, overlap $\Sigma$, strain $\varepsilon$ and grain size $D$ as obtained by WHP and WA, and film thickness $t$ for different spot sizes $d_{\mathrm{b}}\left(v_{\text {scan }}=30 \mathrm{~mm} / \mathrm{s}, \delta=0.1 \mathrm{~mm}, f=100 \mathrm{~Hz}\right)$

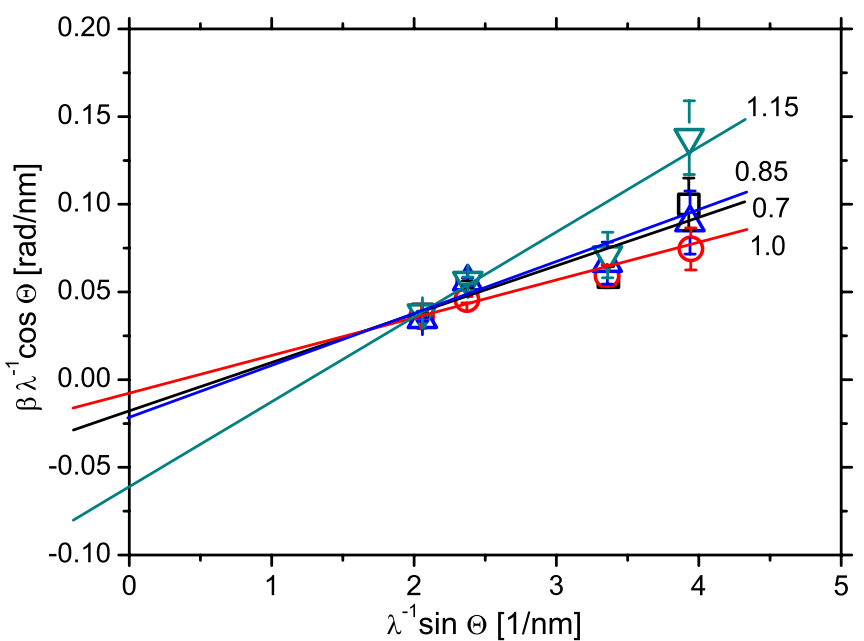

FIGURE 8 WHP plot for different spot diameters. The scan parameters are shown in Table 1

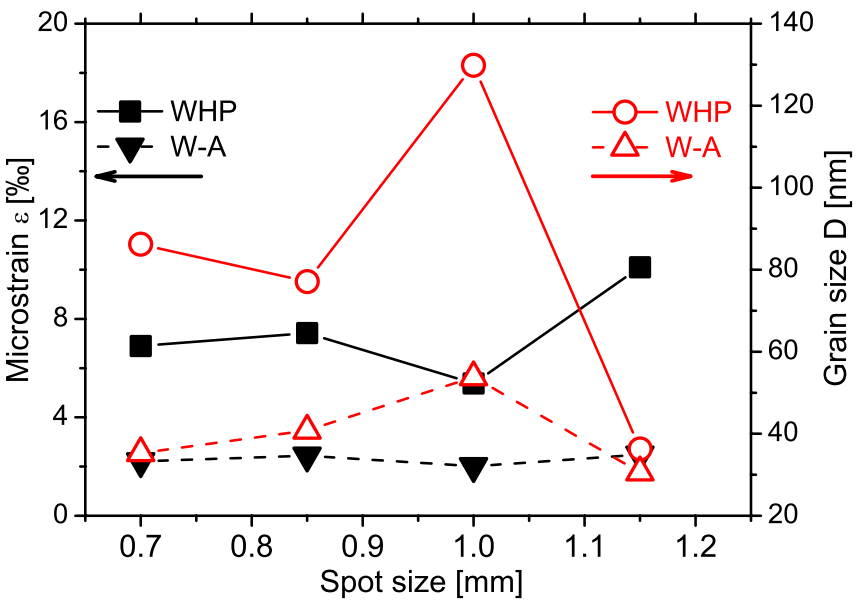

FIGURE 9 Microstrain and grain size as a function of the spot diameter

of the strain and the grain size on the spot diameter. Drastic changes occur between a spot diameter of 0.85 and $1 \mathrm{~mm}$ as seen in Fig. 9. This might be caused by a threshold in the laser intensity for evaporation and nitrogen activation and phase formation dynamics.

Furthermore, the spot size mainly influences the surface roughness. Due to the recoil pressure and the convection effects, the surface quality decreased and melting droplets and cracks resulted. For small spot diameters the evaporation time per pulse increased, so that a stock removal occurred. This should be avoided in order to obtain thick coatings.

4.1.3 Influence of the lateral shift and scan repetition. During meandering of the samples, the lateral shift $\delta$ also influences the processing time and the coating properties. For small shifts, the surface quality decreases as a result of strong energy entry and the remelting effects. Because of convection, the layers become inhomogeneous. Table 5 shows that there is only one optimal value for successful processing. In this case this is $0.1 \mathrm{~mm}$, which is $12 \%$ of the spot size.

The comparison of the results shows that at $\delta=0.1 \mathrm{~mm}$ an optimal ratio between remelting and diffusion exists. It could be assumed that in this case every surface element reaches the 


\begin{tabular}{lccrrrrr}
\hline$\delta$ & $\eta_{\mathrm{t}}$ & $\Sigma$ & $\begin{array}{c}\varepsilon_{\mathrm{WHP}} \\
{[\%]}\end{array}$ & $\begin{array}{r}D_{\mathrm{WHP}} \\
{[\mathrm{nm}]}\end{array}$ & $\begin{array}{c}\varepsilon_{\mathrm{WA}} \\
{[\%]}\end{array}$ & $\begin{array}{c}D_{\mathrm{WA}} \\
{[\mathrm{nm}]}\end{array}$ & $\begin{array}{c}t \\
{[\mu \mathrm{m}]}\end{array}$ \\
\hline 0.20 & 0.952 & 16.7 & 1.760 & 9.8 & 0.523 & 10.4 & 0.50 \\
0.15 & 0.763 & 22.2 & 1.145 & 17.7 & 0.298 & 25.2 & 1.16 \\
0.10 & 0.732 & 33.3 & 0.535 & 126.4 & 0.201 & 53.8 & 1.21 \\
0.05 & 0.662 & 66.7 & 0.675 & 56.5 & 0.224 & 33.6 & 1.29 \\
\hline
\end{tabular}

TABLE 5 Results of the XRD analyses: texture parameter $\eta_{\mathrm{t}}$, overlap $\Sigma$, strain $\varepsilon$ and grain size $D$ as obtained by WHP and WA, and film thickness $t$ for different lateral shifts $\delta\left(v_{\mathrm{scan}}=30 \mathrm{~mm} / \mathrm{s}, d_{\mathrm{b}}=1.00 \mathrm{~mm}, f=100 \mathrm{~Hz}\right)$

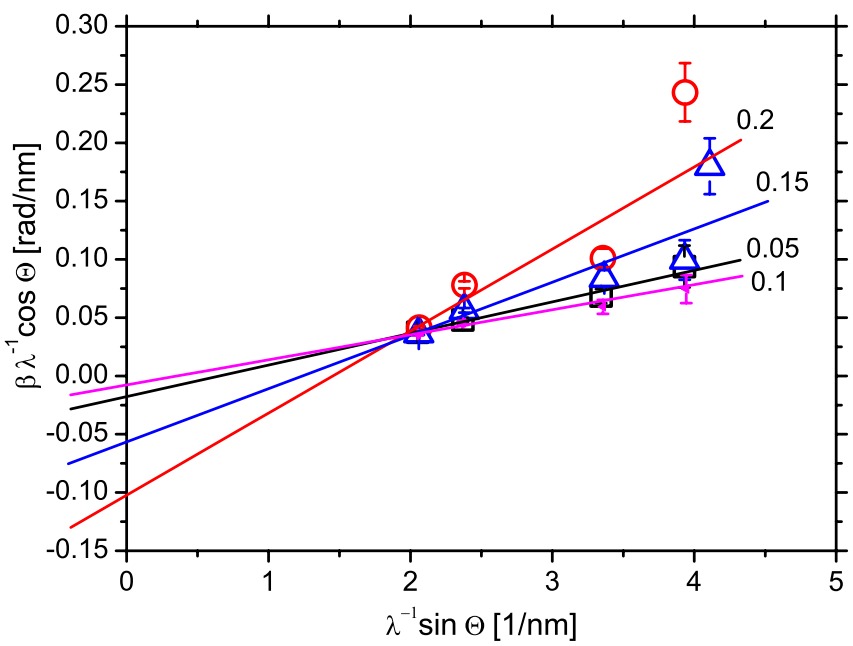

FIGURE 10 WHP plot for different lateral shifts. The scan parameter are shown in Table 1

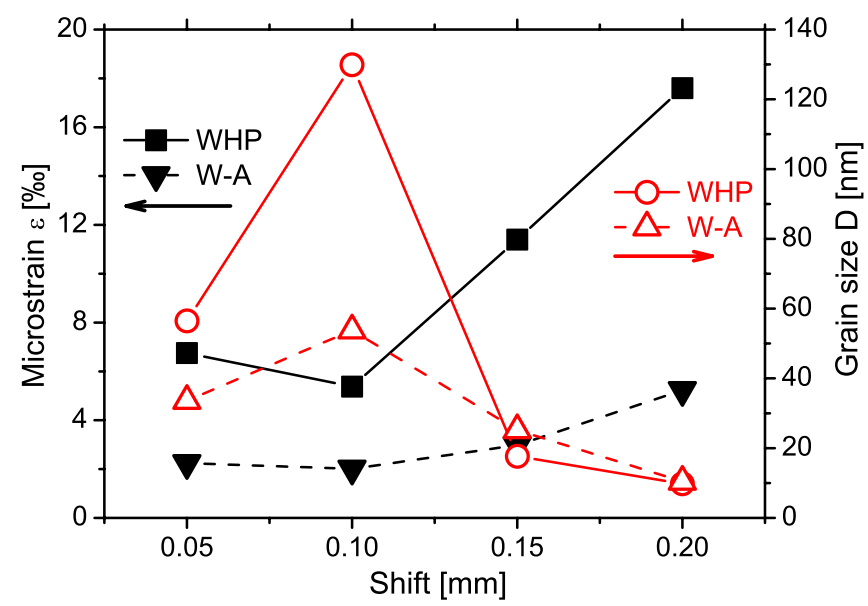

FIGURE 11 Derived microstrain and grain size as a function of the lateral shift

evaporation point. Additionally, all melting droplets and melt remains will be destroyed.

For higher values of the lateral shift, the surface quality decreases rapidly. Due to the larger distance between the scan lines, no complete surface modification could be observed. The remelting effects amplify each other. As a result, the nitrogen distribution on the surface is not homogeneous and ripples develop. As shown in Figs. 10 and 11, this influence is quite strong.

With respect to the proceeding, it is very important to choose the right ratio between the spot size and the shift. To obtain more accurate information, it is necessary to vary the shift in smaller steps, which was not done. Lastly, the scans

\begin{tabular}{llllllll}
\hline$n$ & $\eta_{\mathrm{t}}$ & $\Sigma$ & $\begin{array}{c}\varepsilon_{\mathrm{WHP}} \\
{[\%]}\end{array}$ & $\begin{array}{c}D_{\mathrm{WHP}} \\
{[\mathrm{nm}]}\end{array}$ & $\begin{array}{c}\varepsilon_{\mathrm{WA}} \\
{[\%]}\end{array}$ & $\begin{array}{c}D_{\mathrm{WA}} \\
{[\mathrm{nm}]}\end{array}$ & $\begin{array}{c}t \\
{[\mu \mathrm{m}]}\end{array}$ \\
\hline 1 & 0.732 & 33.3 & 0.535 & 126.4 & 0.201 & 53.8 & 1.21 \\
2 & 0.755 & 66.7 & 0.478 & 227.0 & 0.185 & 55.1 & 1.67 \\
\hline
\end{tabular}

TABLE 6 Results of the XRD analyses: texture parameter $\eta_{\mathrm{t}}$, overlap $\Sigma$, strain $\varepsilon$ and grain size $D$ as obtained by WHP and WA, and film thickness $t$ for different scan repetitions $n\left(v_{\text {scan }}=30 \mathrm{~mm} / \mathrm{s}, d_{\mathrm{b}}=1.00 \mathrm{~mm}, \delta=0.1 \mathrm{~mm}\right.$, $f=100 \mathrm{~Hz}$ )

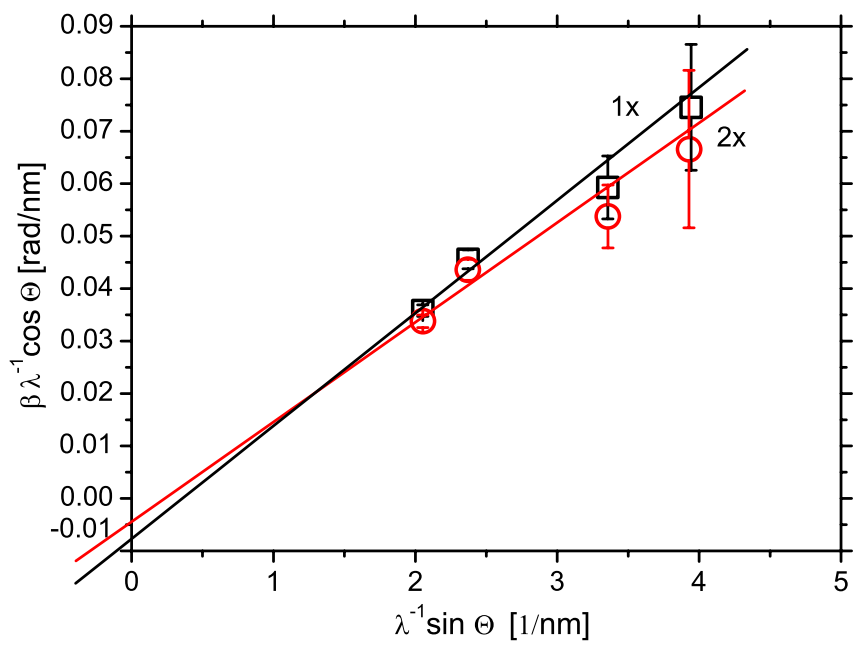

FIGURE 12 WHP plot for one and two scans $n$. The scan parameters are given in Table 1

were repeated. In Table 6 the coating properties are shown for repeating the scan one time.

There are no significant changes in the coating quality when looking at Fig. 12. Only the grain size increases slightly, because in the second scan, titanium nitride will be irradiated and not titanium as in the first one. A comparison of the physical properties like melting and evaporation point explain the weak variations. Additionally, the solubility of nitrogen in the phases is delimited. The melting and evaporation temperatures are much higher than that for pure titanium. In addition, the diffusion coefficient of nitrogen in titanium nitride is much lower than that of titanium.

Therefore, repeated scans do not improve the quality but only increase the processing time.

4.1.4 Correlations. Figures 13 and 14 show the correlation between the grain size and the microstrain. There seems to be a lower limit for the average lattice strain of nearly $1 \%$ and for the maximum lattice strain of about $5 \%$.

Moreover, the grain size is limited to values below $250 \mathrm{~nm}$ (WHP) and $60 \mathrm{~nm}$ (WA).

The limiting physical factors are the inhomogeneous energy distribution during the melting and the different nitrogen content in the depth ranges. It could be assumed that only in regions of about $50 \mathrm{~nm}$ similar conditions exist. This seems to be a limit for such materials processing.

Because of the different procedures and assumptions, the results of the two analyses varied. The WHP analysis only gives the values for the maximum strain. Similar results were 


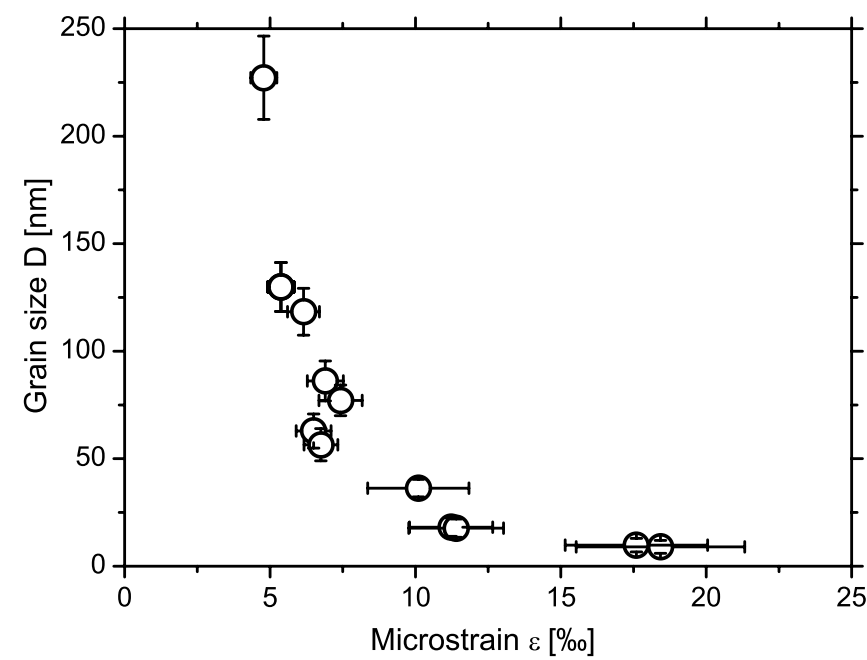

FIGURE 13 Relation between the grain size and the maximum lattice strain (WHP analysis)

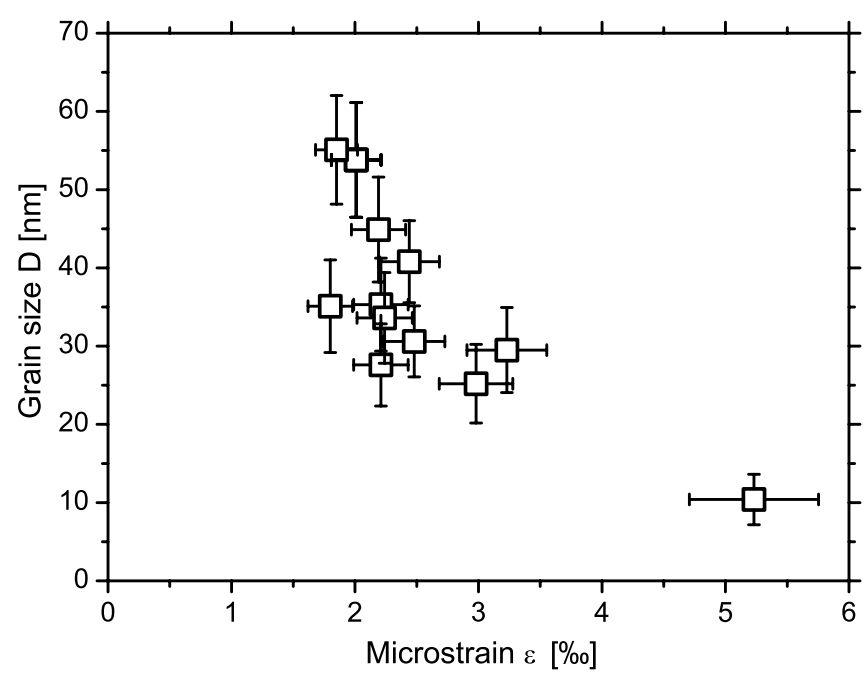

FIGURE 14 Relation between the grain size and the average lattice strain (WA analysis)

observed in the work of Savaloni [34], where the most accurate values were determined by the WA analysis.

A comparison of the XRD analysis results with those of the TEM micrograph in Fig. 15 shows that the results of the WHP plots, for the maximal grain size, are in the same order of magnitude. Also the development of a (200) fiber texture could be observed. The micrograph confirms the results of the $\mathrm{XRD}$ analysis.

\section{2}

\section{The overlap parameter}

The overlap parameter $\Sigma$ was used to simplify the various parameters of the nitriding process. Figures 16 and 17 show that there is an optimal value at about $\Sigma=30$ for the grain size. Large grains improve the macroscopic properties such as hardness.

Due to the short interaction time of the laser beam and the titanium at low overlap values, the crystal growth cannot be supported significantly. None of the important effects like plasma formation and deep melting could be observed. The quality of these coatings is rather poor.

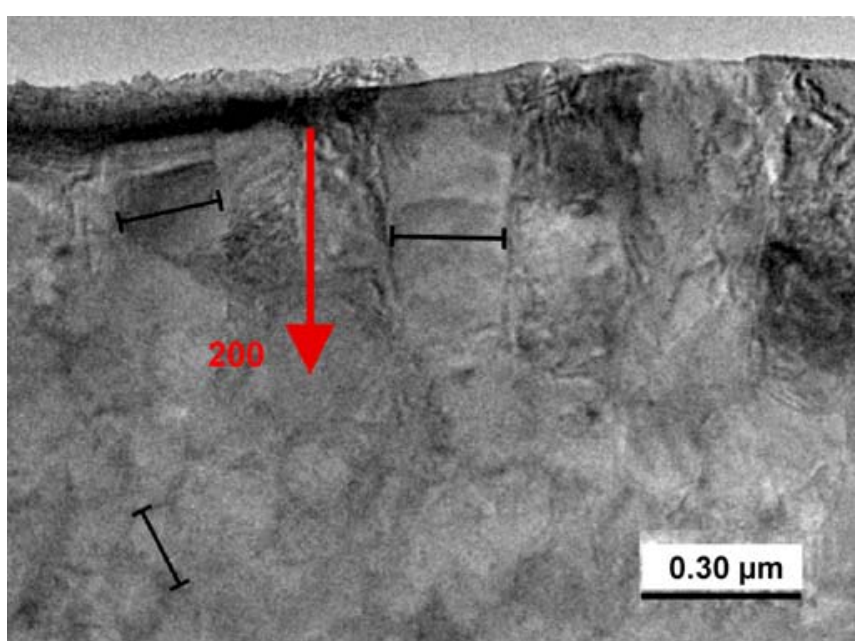

FIGURE 15 TEM-micrograph of sample 2. The preferred orientation and the grain size can be observed

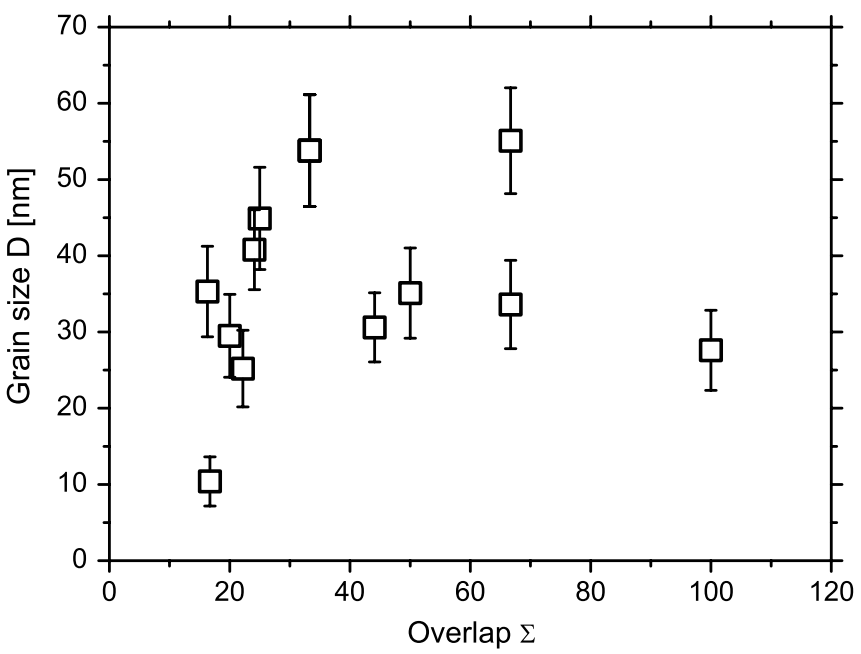

FIGURE 16 Grain size distribution for all the samples and the dependence on the overlap parameter (WA analysis)

However, for high energy input, i.e. high overlap values, the quality also decreases. This is attributed to remelting and convection effects. Figures 18 and 19 show a minimum of lattice strain in the same overlap range. For small overlap parameters up to a value of $\Sigma=30$, the microstrain decreases rapidly to a value of nearly $10^{-3}$. In this range, the strain in the (100) direction is dominant.

After increasing the overlap value above $\Sigma=30$, the strain grows again. There, the (111) strain energy becomes important. During the nitriding, the energy input is so high that the crack development is reduced.

In the case of nitriding with an Nd:YAG laser, an overlap parameter $\Sigma$ between 30 and 35 seems optimal. In this range, the best possible coatings were synthesized. The strain becomes minimal as a result of the equilibrium of the strain energies of the (100) and (111) lattice planes. Under these conditions, the crystallite size increases, resulting in an acceptable quality of the titanium nitride coatings. Furthermore, the nitrogen content in the coatings increases as a result of optimal diffusion conditions. 


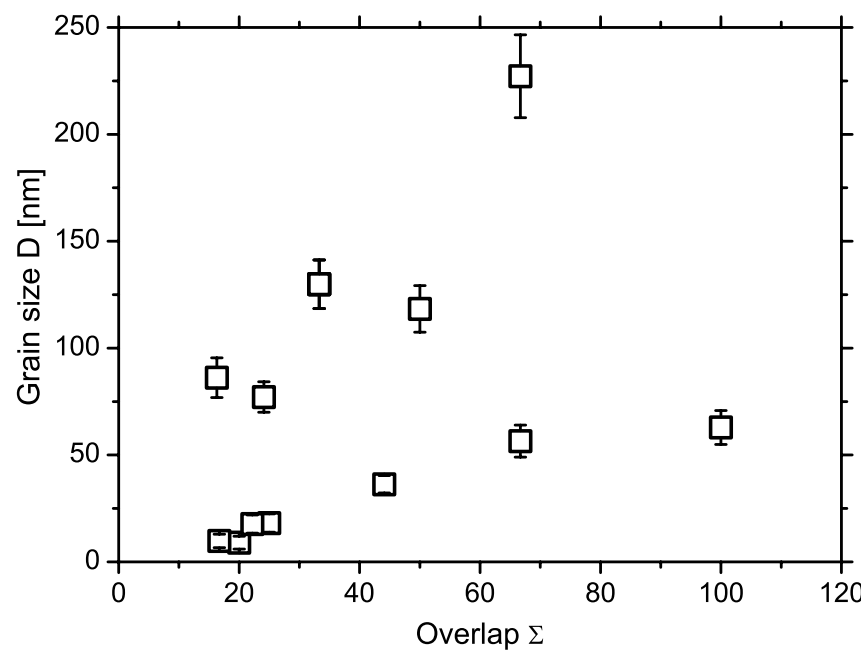

FIGURE 17 Grain size distribution for all the samples and the dependence on the overlap parameter (WHP analysis)

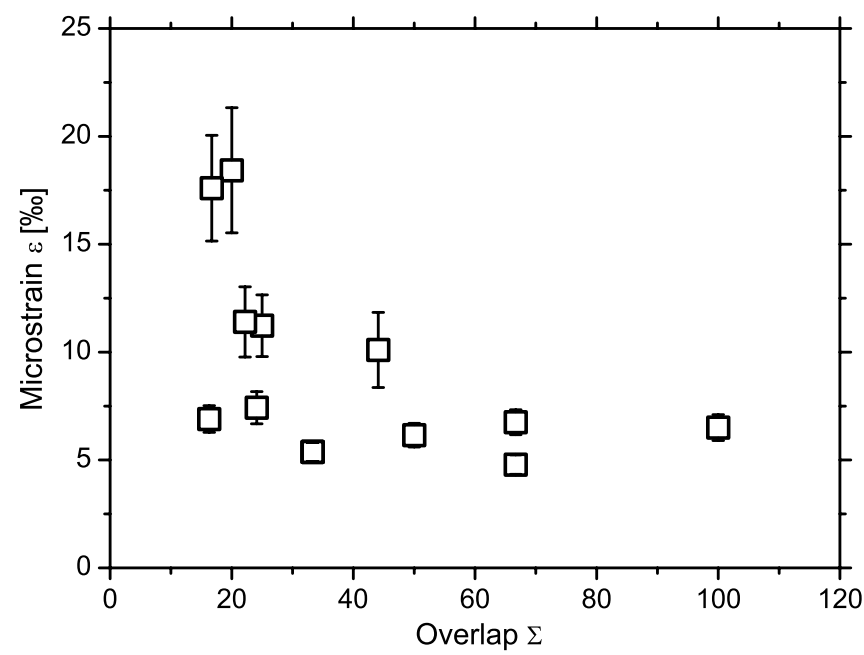

FIGURE 18 Maximal lattice strain for all the samples and the dependence on the overlap parameter (WHP analysis)

\subsection{Preferred lattice orientation and texture development}

The preferred crystallite orientation occurring in direct laser synthesis can be predicted by means of the overlap parameter, too. This also relies on the fact that the Gibbs free energy is minimized. For the theoretical description of the strain development, the model of Zhao [13] was used. The orientation is determined by the competition between the surface energy $S_{h k l}$, the strain energy $U_{\text {surface }(h k l)}$ for every plane and the energy $E_{h k l}$ induced by external forces (negligible here). The sum of these three terms is called the overall energy $W_{h k l}$. The orientation of a grain will develop in such a way that this overall energy is minimized.

$W_{h k l}=S_{h k l}+U_{\text {surface }(h k l)}+E_{h k l}$
$U_{h k l}=\varepsilon_{h k l}^{2} e_{h k l}\left(1-v_{h k l}\right)$
$U_{\text {surface }(h k l)}=U_{h k l} t$.

In relation to the coatings presented here, the term $E_{h k l}$ can be neglected because the induced strain is dominant.

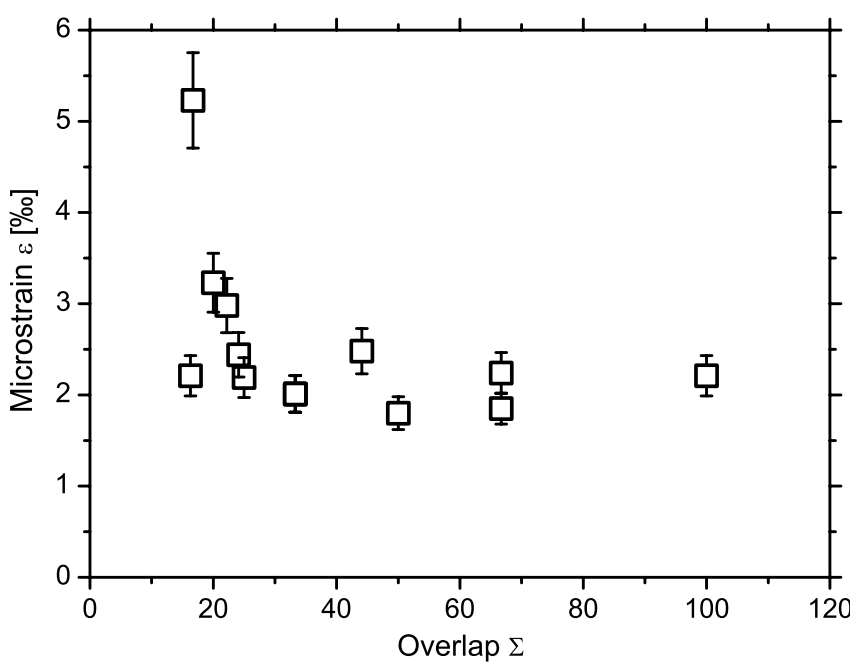

FIGURE 19 Average lattice strain for all the samples and the dependence on the overlap parameter (WA analysis)

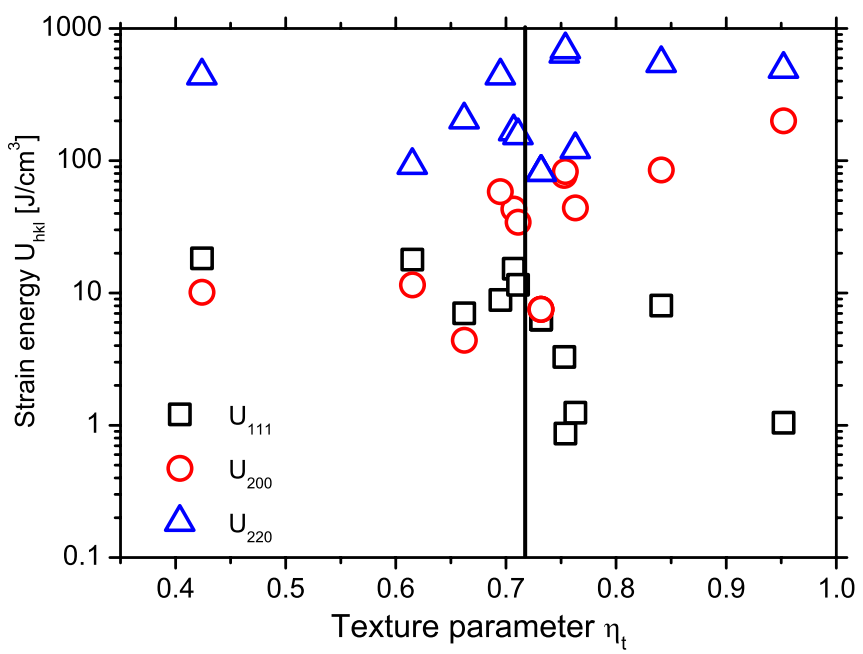

FIGURE 20 Strain energy $U_{h k l}$ for the (200), (111) and (220) lattice planes (Voigt analysis) as a function of the texture parameter

The surface energy for every lattice plane was calculated in the work of Zhao [13]. In the case of cubic titanium nitride, the values are $S_{200}=4.94 \mathrm{~J} / \mathrm{cm}^{2}, S_{220}=6.99 \mathrm{~J} / \mathrm{cm}^{2}$ and $S_{111}=8.53 \mathrm{~J} / \mathrm{cm}^{2}$. The ratio of the different lattice planes is $1: 1.41: 1.73$ so that in the case of less strain, the (200) direction will be preferred.

The strain energy for every lattice plane is shown in Fig. 20. $U_{h k l}$ was calculated by (14)). For this, the lattice strain $\varepsilon_{h k l}$ was measured by the single line Voigt method. Young's moduli $e_{h k l}$ were taken from the work of Meng [38], who proposed values for the (200) and the (111) lattice planes. They are identified as $e_{200}=476.5 \mathrm{GPa}$ and $e_{111}=396.4 \mathrm{GPa}$. Further, the values of the Poisson ratio are given as $\nu_{200}=0.159$ and $v_{111}=0.216$. Because there are no data available for the (220) plane, they were calculated by the ratios of the Young's moduli shown in the work of Zhao [13], i.e. a value of $438 \mathrm{GPa}$. The Poisson ratio $\nu_{220}=0.2$ was taken as an average value. This value was used in the publications of Valvoda [39] and Pelleg [14]. Figure 20 shows that $U_{220}$ is much higher than the other one, thus only (111) and (200) orientations could be observed. 


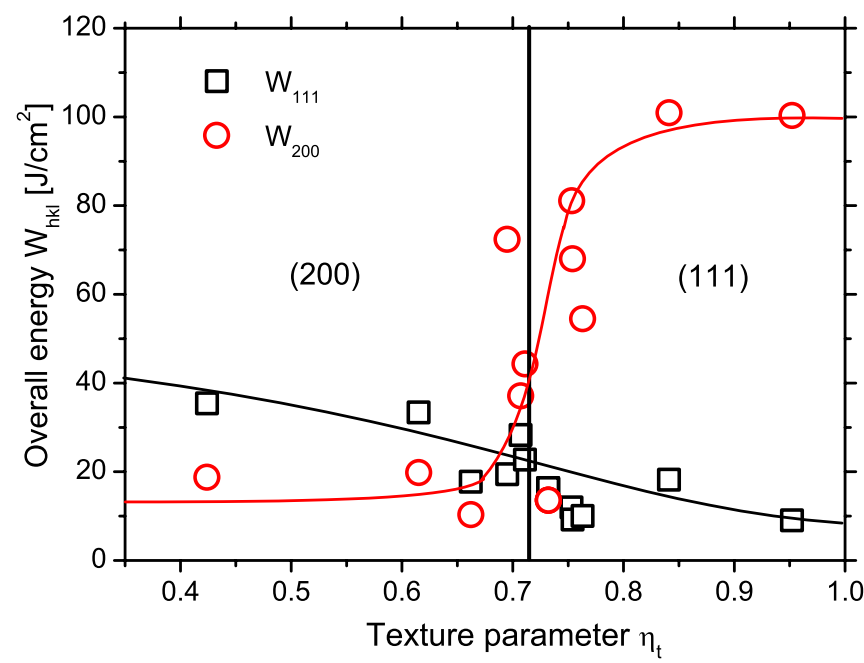

FIGURE 21 Overall energy $W_{h k l}$ for the (200) and (111) lattice planes (Voigt analysis). The orientation of the samples is shown

Finally, the decisive factor for the orientation is the competition of the overall energy $W_{h k l}$. Figure 21 represents the calculated values for the nitrided samples. The strain energy of every plane $U_{\text {surface }(h k l)}$ was calculated with (14) by means of the layer thicknesses shown in Tables 3-6. Theoretically, the equilibrium of the lattice strain is at a texture parameter $\eta_{\mathrm{t}}$ of 0.72 . By relating to the experiments, similar results could be observed. The large fluctuations were determined by the statistical errors and the experimental and theoretical drawbacks (errors) of the Voigt analysis model.

The dependence of the orientation as a function of the overlap parameter is shown in Fig. 22. For a value of $\Sigma=30$ 35 the orientation changes. The strain energy is the most important factor. For example, in the case of sample 1 the value of $U_{\text {surface(111) }}$ is about $40 \mathrm{~J} / \mathrm{cm}^{2}$. This is five times higher than the surface energy. On the basis of the overlap concept, the orientation of the lattice during laser nitriding can be controlled.

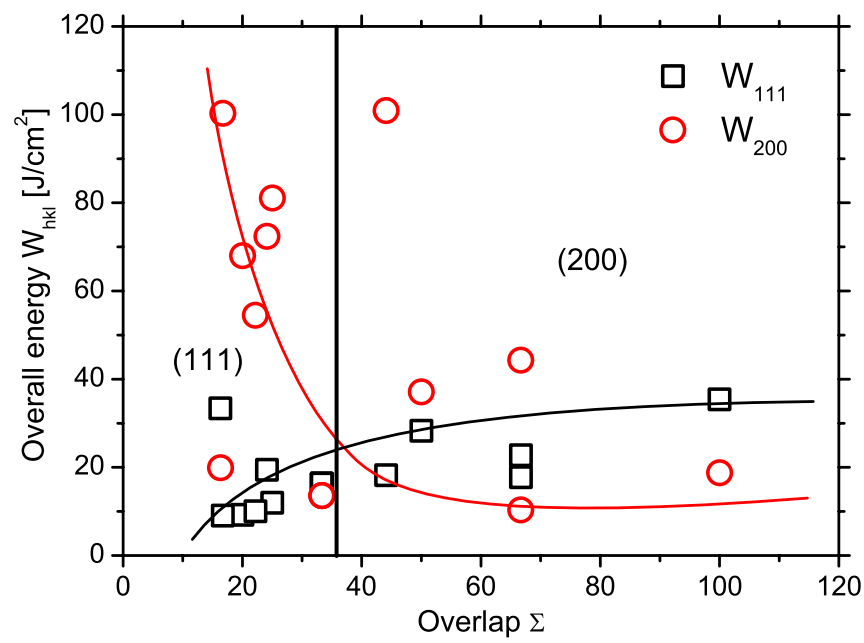

FIGURE 22 Overall energy $W_{h k l}$ for the (200) and (111) lattice planes (Voigt analysis) in relation to the overlap parameter. The orientation of the samples is shown

\section{5}

\section{Conclusions}

$\mathrm{TiN}_{x}$ coatings with thicknesses in the micron range were synthesized by direct laser nitriding of titanium. A detailed analysis by X-ray diffraction could resolve strain, grain size, and texture in the resulting TiN coatings. The results can be correlated to the processing parameters and the coating properties. For optimal parameters, the strain could be reduced down to a value of 0.001 and the maximum achievable grain size is around $100 \mathrm{~nm}$. The grain size is limited by convection, diffusion, and heat transfer. The optimal coating properties were achieved with an overlap parameter of $\Sigma=33.3$.

The preferred orientation in the coatings could be related to the strain distribution and thus to the processing parameters. Finally, it seems possible to predict strain, grain size, and preferred orientation for $\mathrm{Nd}$ :YAG laser nitriding of titanium by means of the overlap parameter.

ACKNOWLEDGEMENTS This work has been supported by the Deutsche Forschungsgemeinschaft (DFG grant Scha 632/4). Peter Schaaf gratefully acknowledges the support of a Heisenberg fellowship (DFG Scha 632/11).

OPEN ACCESS This article is distributed under the terms of the Creative Commons Attribution Noncommercial License which permits any noncommercial use, distribution, and reproduction in any medium, provided the original author(s) and source are credited.

\section{REFERENCES}

1 A. Bloyce, P.H. Morton, T. Bell, Surface engineering of titanium and titanium alloys (ASM Int., Member/Customer Service Center, Materials Park, 1994)

2 H.C. Man, Z.D. Cui, T.M. Yue, F.T. Cheng, Mater. Sci. Eng. A 355, 167 (2003)

3 H.J. Spies, P. Schaaf, F. Vogt, Materialwiss. Werkst. 29, 588 (1998)

4 P. Schaaf, A. Emmel, C. Illgner, K.P. Lieb, E. Schubert, H.W. Bergmann, Mater. Sci. Eng. A 197, L1 (1995)

5 P. Schaaf, Prog. Mater. Sci. 47, 1 (2002)

6 P. Schaaf, M. Han, K.-P. Lieb, E. Carpene, Appl. Phys. Lett. 80, 1091 (2002)

7 E. Carpene, P. Schaaf, Appl. Phys. Lett. 80, 891 (2002)

8 E. Carpene, P. Schaaf, Phys. Rev. B 65, 224111 (2002)

9 E. Carpene, M. Shinn, P. Schaaf, Appl. Phys. A: Mater. 80, 1707 (2005)

10 H. Xin, S. Mridha, T.N. Baker, J. Mater. Sci. 31, 22 (1996)

11 D. Höche, M. Shinn, J. Kaspar, G. Rapin, P. Schaaf, J. Phys. D 40, 818 (2007)

12 U.C. Oh, J.H. Je, J. Appl. Phys. 74, 1692 (1993)

13 J.P. Zhao, X. Wang, Z.Y. Chen, S.Q. Yang, T.S. Shi, X.H. Liu, J. Phys. D 30, 5 (1997)

14 J. Pelleg, L.Z. Zevin, S. Lungo, N. Croitoru, Thin Solid Films 197, 117 (1991)

15 T.B. Massalski, H. Okamoto, P.R. Subramanian, L. Kacprzak (Eds.), Binary Alloys Phase Diagrams (ASM Int., Materials Park, 1990)

16 W.-E. Wang, J. Alloys Compd. 233, 89 (1996)

17 L. Vegard, Z. Phys. 5, 17 (1921)

18 S. Nagakura, T. Kusunoki, F. Kakimoto, Y. Hirotsu, J. Appl. Cryst. 8, 65 (1975)

19 A.R. Stokes, Proc. Phys. Soc. 61, 382 (1948)

20 S. Krumm, Acta Universitatis Carolinae Geologica 38, 253 (1994)

21 S.A. Howard, R.L. Snyder, J. Appl. Cryst. 22, 238 (1989)

22 T.H. de Keijser, J.I. Langford, E.J. Mittemeijer, A.B.P. Vogels, J. Appl. Cryst. 15, 308 (1982)

23 J. Langford, J. Appl. Cryst. 11, 10 (1978)

24 M. Wojdyr, FITYK-0.7.7; A curve fitting and data analysis program (2004) http://www.unipress.waw.pl/fityk/

25 P. Scherrer, Nachr. Ges. Wiss. Göttingen 26, 98 (1918)

26 A.J.C. Wilson, Proc. R. Soc. London Ser. A 181, 360 (1943)

27 A.R. Stokes, A.J.C. Wilson, Proc. Phys. Soc. 56, 174 (1944)

28 G.K. Williamson, W.H. Hall, Acta Metall. 1, 22 (1953) 
29 B.E. Warren, B.L. Averbach, J. Appl. Phys. 21, 595 (1950)

30 B.E. Warren, X-ray Diffraction (Dover Pub., New York, 1990)

31 C.N.J. Wagner, E.N. Aqua, Adv. X-ray Anal. 7, 46 (1964)

32 P. Schaaf, G. Rixecker, E. Yang, C.N.J. Wagner, U. Gonser, Hyperfine Interact. 94, 2239 (1994)

33 C.N.J. Wagner, E. Yang, M.S. Boldrick, Adv. X-ray Anal. 35, 585 (1992)

34 H. Savaloni, M. Gholipour-Shahraki, M.A. Player, J. Phys. D Appl. Phys. 39, 2231 (2006)
35 D.R. McKenzie, Y. Yin, W.D. McFall, N.H. Hoang, J. Phys.: Condens. Matter 8, 5883 (1996)

36 T. Lee, K. Ohmori, C.-S. Shin, D.G. Cahill, I. Petrov, J.E. Greene, Phys. Rev. B 71, 144106 (2005)

37 PDF2 Database 1997 PCPDFWIN 1.30, Joint Committee for Powder Diffraction Studies (JCPDS)-ICDD

38 W.J. Meng, G.L. Eesley, Thin Solid Films 271, 108 (1995)

39 V. Valvoda, J. Musil, Thin Solid Films 149, 49 (1987) 